\title{
Optimization algorithm and implementation of 8PSK modulation
}

\author{
Chang-Ying $\mathrm{Li}^{1, \text { a }}$ and Hua-Hai Qiu ${ }^{2, b, *}$ \\ ${ }^{1}$ Wenhua College, Wuhan, China \\ ${ }^{2}$ Wuhan Textile University, Wuhan, China \\ Email: ${ }^{a}$ lichangying0420@163.com, ${ }^{b}$ qiuhuahai2006@163.com
}

\begin{abstract}
Modulation technology is very important in the field of communication. A good modulation technology not only can save spectrum resources but also can provide a good channel performance. This paper is based on TCM+8PSK modulation demodulation principle of waveform research and analysis, which aims at analysis and design of the key technologies of 8PSK optimization algorithm and implementation, and the performance of the model simulation at the same time. The simulation results show that the TCM+8PSK modulation and demodulation techniques are used to obtain high gain in $225-512 \mathrm{MHzUHF}$ frequency band. At the same time, it has good performance in anti-multipath equalization, and the optimization algorithm has good performance in high speed digital transmission and anti- interference.
\end{abstract}

Keywords: UHF; TCM+8PSK; Data Transmit; Multipath Delay; Adaptive Equalization; RS Coding.

\section{Introduction}

In the digital signal modulation, 8PSK is the most commonly used digital signal modulation mode, it has high spectrum utilization and strong anti-interference ability. Compared with other modulation signals, such as FSK, PSK, and other modulated signals, the band is less, and the adjacent channel interference is smaller[1].

Trellis Code Modulation (TCM) technology [2]is first put forward in 1982 by UugerBoeck, joint design of coding and modulation for TCM, compared with the traditional non-coded multilevel modulation, the significant coding gain is obtained by extending the signal set of the multilevel level phase without the extension of the bandwidth.

In recent years, as the technology continues to mature, TCM technology is widely used in data transmission system, so the technology is particularly suitable for application in the UHF band to data transmission business based communications equipment. 
This paper is based on the 225-512MHzUHF band, combine the TCM+8PSK organicly, using MATLAB simulation tool for its modulation and demodulation mode in the AWGN channel and QPSK modulation coding method for performance comparison analysis, the performance of TCM+8PSK is better than QPSK when $10^{-2}$ is lower than BER, and the gain of QPSK is about $3 \mathrm{~dB}$ when BER is $10^{-5}$, in the meantime it also has good performance in anti -multipath equalization and so on.

\section{Principle Design of Waveform Overall Scheme}

\subsection{Principle of digital transmission waveform based on TCM+8PSK}

This paper puts forward solution for TCM+8PSK modulation demodulation technique. TCM is used for segmentation principle, combines convolutional coding and modulation, designs the coding sequence with minimum Euclidean distance, thereby the soft output Viterbi decoder can obtain the ber performance of the optimal, it is a kind of methods which are frequently used in the high-speed data transmission[3].

Using muti- band modulation, which means each data symbol can carry multiple bits of information. In this way, the transmission rate of bits can be improved effectively without changing the speed. However, due to the expansion of the whole signal constellation, the Euclidean distance between the signal points is reduced, which leads to the error rate of the system is improved greatly. In order to make the system error rate to meet the system requirements, we must use efficient channel coding technology. TCM coded modulation is a kind of effective coding way, which is designed by combinating with coding and modulation, in this way, we can enhance system performance by 3 to $6 \mathrm{~dB}$ without reducing the transmission rate of information and increase system bandwidth.

\subsection{The structure design of transmitter and receiver}

Simulation model of a transmitter and a receiver structure diagrams, as shown in figure 1, figure 2, as is shown in visible in addition to the digital transmission of $\mathrm{TCM}+8$ PSK modulation demodulation way, RS coding and decoding with excellent anti burst interference performance is also added[4], so the waveform have the characteristics of high speed transmission and strong anti-interference.

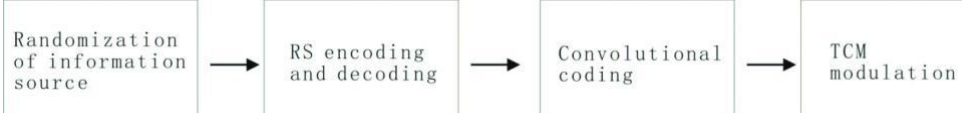

Fig. 1 The structure of transmitter in physical layer 


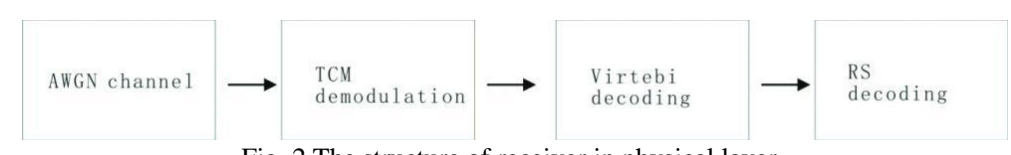

Fig. 2 The structure of receiver in physical layer

Constructing the simulation model platform by Matlab, it is mainly made up of Reed Solomon channel encoding and TCM+8PSK encoding, as follows:

1) The Reed Solomon channel encoding is made up of three parts, one of them based on GF generating polynomial; the other two is RS coding module and RS decoding module. Each symbol in simulation RS encoding and decoding that we use decimal to represent, the rate of each symbol is 8 bit according to the project, RS code is also 8 bit which uses decimal to represent[5].

2) The TCM+8PSK part. Convolutional coding for each RS data block which is the same as the convolutional code structure of 3G WiMAX 802.16-(2.1.7)convolutional code, the coding rate is $1 / 2$, Constraint length is 7 , and then to code with following polynomial, as folows:

$$
G_{1}=133_{\text {OCT }} G_{2}=171_{\text {OCT }}
$$

\section{Analysis and Design of the Key Technology}

\subsection{Design of TCM+8PSK modulation and demodulation}

When modeling and simulation, we combine with convolutional coding and 8PSK perfectly. To ensure longest Euclidean distance, we select the corresponding point of the 8PSK constellation points on the convolution code.

When FPGA is in implementation, the optimal state of the $(2,1,7)$ convolutional code and 8PSK modulation combined with the TCM encoder is used here. We need to pay attention to the state transfer settings and statistics in implementation, and Viterbi decoding uses soft decision method to improve the decoding accuracy, combining with the transmitter and receiver system timing synchronization requirements, which will increase the different frames for timing synchronization of temporal order judgment and control.

\subsection{Design and implementation of equalization technology}

The above theory and the simulation results show that inserting a kind of tunable filter can correct and compensate the characteristics of the system and reduce the effect of inter symbol interference ,The compensation effect of the filter is the equalizer in the communication system, adaptive equalization technology can track the change of the channel dynamically, and adjust the parameters of the 
equalizer in time, and select the appropriate decision criterion according to the application scene. The main implementation of adaptive equalizer is transversal filter, which is mainly composed of tapped delay line, weighted coefficient multiplier and adder.

For the calculation of similar complex multiplier and clock cycle (frequency), multi rate filter(CIC and FIR half band),you can try to reuse the current platform FPGA chip comes with IP core, it can be easy to achieve hardware circuit directly.

\section{Performance Simulation Analysis}

\subsection{Simulation analysis based on TCM+8PSK characteristics}

\subsubsection{The selection of modulation mode}

The performance of TCM+8PSK and QPSK two modulation coding methods are compared, and the simulation results are shown in the figure 3:

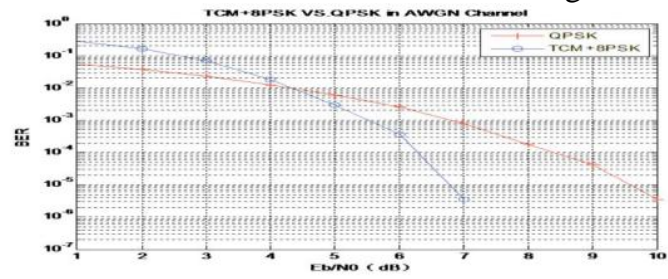

Fig. 3 TCM+8PSKVS.QPSK Performance comparison chart

As is known from the figure, when the BER is lower than 10-2, the performance of TCM+8PSK is better than QPSK. When the BER is 10-5, the performance is also better than that of QPSK, at this moment, the gain of which is $3 \mathrm{~dB}$ better than that of QPSK. Therefore, this program selects TCM+8PSK modulation.

\subsubsection{Selection of TCM+8PSK production polynomial}

The convolution code $(2,1,7)$ is used to generate polynomial in TCM+8PSK. The constraint length of the convolutional code is 7 , the state number is 64 , and the polynomial coefficients are 133 and 171 .

Generally speaking, convolutional codes are used to increase the minimum distance by increasing the constraint length, which is used to improve the system reliability. The more the state of the TCM, the performance is also more advantages. The three kinds of generating matrices $G$ (i.e. generating 
polynomial) of different convolutional codes are simulated and verified, the three kinds of production polynomials are as follows:

$$
(2,1,4), \mathrm{G}=([4],[152])
$$

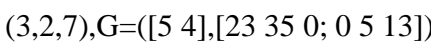

$$
\begin{aligned}
& (2,1,7), \mathrm{G}=([7],[133171])
\end{aligned}
$$

We can see that the third generation matrix $\mathrm{G}$ has the best performance, so the generation polynomial of the scheme TCM selects $(2,1,7)$.

\subsubsection{Simulation key point}

It mainly reflected in the RS code, here is the following binomial CDF (cumulative distribution function) formula

$$
y=F(x \mid n, p)=\sum_{i=0}^{x}\left(\begin{array}{l}
n \\
i
\end{array}\right) p^{i}(1-p)^{n-i} I_{(0,1 \ldots n)}(i)
$$

The physical meaning of $\mathrm{x}, \mathrm{n}$, and $\mathrm{p}$ represented by the RS code is the supervisory position of $\mathrm{n}-\mathrm{k}$ code, the code word length is $\mathrm{N}$, and the percentage of anti-interference. For the results of $\mathrm{Y}$, Known in the $\mathrm{N}$ independent tests, for each test event, in order to obtain the successful probability of $\mathrm{P}$, which can be observed (i) $\mathrm{x}$ times of the cumulative probability of success. Furthermore $T_{(0,1 \ldots n)}(l)$ defines the value range of $\mathrm{x}$ is $0,1, \ldots \mathrm{N}$.

\subsection{The results of Performance simulation}

Simulation results for various transmission data rates are shown in Fig. 4, The $256 \mathrm{Kbps}$ data transmission waveform is divided into two types, one is RS code and the other one not. In additional to this, other rates are RS coding.

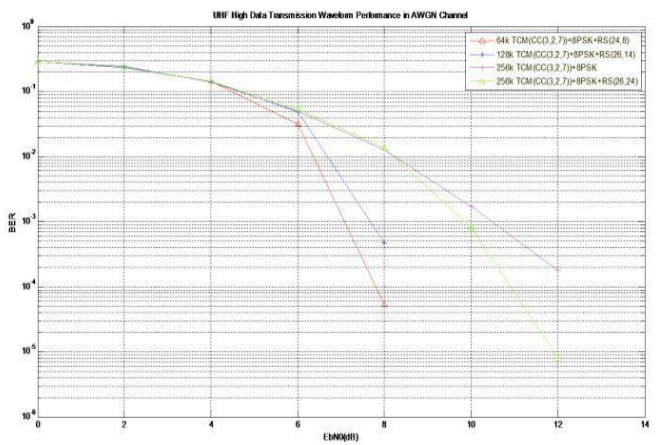

Fig. 4 Demodulation performance at different rates 


\section{Conclusion}

This paper is based on TCM+8PSK modulation demodulation principle of waveform research and analysis, it aims at analysis and design of the key technologies of 8PSK optimization algorithm and implementation, and the performance of the model simulation at the same time. The simulation results show that the TCM+8PSK modulation and demodulation techniques are used to obtain high gain in $225-512 \mathrm{MHzUHF}$ frequency band, at the same time, it has good performance in anti-multipath equalization, and the optimization algorithm has good performance in high speed digital transmission and anti- interference.

\section{References}

1. J.B.Ande:rson, T.Aulin and C.E.Sundberg, "Digital Phase Modulation”,New York, Plenum Press, 1986.

2. G.Ungerboeck, "Channel coding with multilevel phase sigals"IEEE Trans.Inform. Theory, vol.IT-28, pp.55-67, Jannuary 1982.

3. F. K. Huang, J. W. Chen and S. Q. Lou. Modern digital signal processing. Beijing: Electronic Industry Press, 2003.

4. P. L. Qiu. Information theory and encoding. Beijing: Higher Education Press, 2003.

5. S. Haykin. Adaptive filter theory (Fourth Edition). Translated by B. Y. Zheng et al. Beijing: Electronic Industry Press, 2001. 